\title{
Analysis of Risk Factors for Postoperative Delirium in Patients with Esophageal Cancer
}

\author{
Menglu Zhang, Xiaozhi Bai, Yuting Zhang, Xiaojuan Xie* \\ College of Clinical Medicine of Henan University of Science and Technology/ The First Affiliated Hospital of Henan University of \\ Science and Technology, Luoyang 471003, Henan, China \\ Email: 13693818030@163.com
}

\begin{abstract}
Objective - To investigate the occurrence and risk factors of postoperative delirium in patients with esophageal cancer. Methods - A review of 203 patients undergoing elective surgery for esophageal cancer from April 2019 to December 2020, including 122 males and 81 females. The incidence of postoperative delirium were evaluated through the use of the CAM and divided into delirium group (Group P) and non-delirium group (Group N) according to whether postoperative delirium occurred. Possible confounders and clinically important factors were included in the univariate analysis, and all variables with P-values less than 0.05 in the univariate analysis were included in the multivariate models. Results - A total of 38 cases (18.7\%) of postoperative delirium occurred in 203 patients. Group P was significantly older than Group N $(\mathrm{P}=0.000<0.01)$. The Patient Health Questionnaire $9(\mathrm{PHQ}-9)$ scores $(\mathrm{P}=0.01<0.05)$ and the FRAIL Scale scores of Group $\mathrm{P}$ were significantly higher than those of the $\mathrm{N}$ group $(\mathrm{P}=0.001<0.05)$. The educational level $(\mathrm{P}=0.001<0.01)$ and Mini-Mental State Examination (MMSE) scores $(\mathrm{P}=0.004<0.01)$ of Group $\mathrm{P}$ were significantly lower than those of Group $\mathrm{N}$. The use rate of sevoflurane in Group $\mathrm{P}(\mathrm{P}=0.011<0.05)$, the incidence of intraoperative hypotension $(\mathrm{P}=0.002<0.05)$, and the rate of Porphyromonas gingivalis infection $(\mathrm{P}=0.012<0.05)$ were higher than those in Group $\mathrm{N}$. Multivariate logistic regression analysis showed that age $(\mathrm{OR}=1.295,95 \% \mathrm{CI} 1.125 \sim 1.490)$ and Porphyromonas gingivalis infection $(\mathrm{OR}=2.898,95 \% \mathrm{CI}$ 1.055 7.959) were independent risk factors for postoperative delirium in patients with esophageal cancer. Conclusion age and Porphyromonas gingivalis infection may be the independent risk factors for postoperative delirium in patients with esophageal cancer.
\end{abstract}

Keywords: postoperative delirium, esophageal cancer, risk factors

With the aging of the global population and the gradual increase of surgical opportunities for elderly patients, the occurrence of postoperative delirium (POD) has attracted more and more attention from various clinical disciplines. POD is one of the common central nervous system complications after surgery, mainly manifested as confusion, anxiety, personality and social cognitive ability changes, memory impairment, etc., which can seriously reduce the quality of life of patients ${ }^{[1]}$. Studies have shown that the incidence of POD in cardiothoracic surgery can be as high as $57 \%$. Esophageal cancer is one of the common malignant tumors, and there is still a lack of research on the risk factors of POD in patients with esophageal cancer after surgery. In this study, 203 patients who underwent elective radical resection of esophageal cancer from April 2019 to December 2020 were selected to count the incidence of POD after esophageal cancer and their related factors before, during and after surgery were analyzed. This study aimed to look for independent risk factors for POD and provide ideas for the prevention or treatment of POD.

\section{Materials and methods}

\subsection{General information}

This study was approved by the Ethics Committee of the First Affiliated Hospital of Henan University of Science and Technology, and the patient or his family members have signed an informed consent form. Select patients who underwent elective surgery for esophageal cancer from April 2019 to December 2020. Inclusion criteria: patients who have a clear clinical diagnosis and plan to undergo esophageal cancer surgery, volunteer to participate in this study and have good compliance. Exclusion criteria: clear history of neurological disease or mental illness, current use of anti-anxiety, depression and other psychotropic drugs, severe heart, liver, and kidney dysfunction, severe hearing or visual impairment. Rejection criteria: those who changed the operation method during the operation or did not undergo the operation. 


\subsection{Methods of anesthesia}

All patients were injected intramuscularly with $0.5 \mathrm{mg}$ penehyclidine hydrochloride before entering the room, and underwent routine monitoring after entering the room, including ECG, BP, SPO2, and BIS, and invasive arterial blood pressure testing if necessary. If there was no contraindication, an epidural puncture was performed before the induction of anesthesia, the degree of analgesia and the level of anesthesia were evaluated, and the epidural puncture was confirmed successfully, then general anesthesia was induced. The remaining patients underwent general anesthesia induction. During the operation, all patients received intravenous general anesthetics, propofol, remifentanil, and atracurium besylate for anesthesia maintenance. Among them, patients with general anesthesia combined with epidural anesthesia were supplemented by an epidural catheter every 50 minutes by $0.2 \%$ Ropivacaine $5 \mathrm{ml}$. During the operation, maintain PETCO2 at 35-40 $\mathrm{mmHg}$, BIS at 40-60, and blood pressure fluctuations within $30 \%$ of the baseline.

\subsection{Observation indicators}

Record the patient's preoperative information, including: gender, age, education level, history of hypertension, history of diabetes, history of cardiovascular and cerebrovascular diseases, history of previous surgery, ASA classification, and Patient Health Questionnaire (PHQ-9) score, Mini Mental State Examination (MMSE) score, the FRAIL Scale score, serum albumin, whether it is infected with Porphyromonas gingivalis (Porphyromonas gingivalis, P.g). Intraoperative information includes: whether to combine epidural nerve block, whether to use sevoflurane during surgery, intraoperative blood loss, whether intraoperative blood transfusion, whether intraoperative hypotension occurs. Postoperative information: pain numerical scale (numeric rating scale, NRS) score, etc.

The diagnosis of POD was judged by the Confusion Assessment Method (CAM). The evaluation of POD included 4 core symptoms: (1) acute attack or repeated symptom fluctuations; (2) attention deficit; (3) confusion of thinking; (4) changes in the level of consciousness. If the patient has symptoms (1) and (2), and at least one of (3) or (4) is positive, it can be diagnosed as POD. The patients were evaluated for POD at 1 day and 3 days after surgery.

\subsection{Statistical methods}

Use SPSS21.0 statistical software to process the corresponding data. Non-rank count data was expressed by percentage $(\%)$ or rate, count data used $\chi 2$ test or Fisher's exact probability method, grade count data used Kruskal-Wallis rank sum test. In univariate analysis, independent variables with statistically significant differences were brought into the logistic regression equation. $\mathrm{P}<0.05$ indicated a statistical difference.

\section{Results}

\subsection{General conditions}

Among 203 patients, 38 cases (18.7\%) had POD, 26 cases occurred on the first day after surgery, 12 cases occurred on 2-3 days after surgery; 165 cases (81.3\%) had no POD. Comparison of the two groups of patients: age, education level, FRAIL value, PHQ9 value, MMES score, P.g infection were statistically significant $(\mathrm{P}<0.05)$. Comparison of intraoperative and postoperative information between the two groups: the use of sevoflurane and intraoperative hypotension were statistically significant $(\mathrm{P}<0.05)$.

\subsection{Multivariate logistic regression analysis of postoperative delirium in patients with esophageal cancer}

Taking POD as the dependent variable, the factors with statistical differences in general data were included in the multivariate logistic regression analysis. The results showed that advanced age (OR=1.096, 95\%CI 1.038 1.158), P.g infection $(\mathrm{OR}=3.246,95 \% \mathrm{CI}$ 1.587 6.641) were independent risk factors for POD in patients with esophageal cancer $(\mathrm{P}<0.05)$.

\section{Discussion}

One of the mechanisms of POD is the central inflammatory response and neuronal damage caused by anesthesia and surgery itself, which is also an important factor in its pathophysiology. Surgery and anesthesia stimulation can activate the peripheral innate immune system, promote the release of peripheral inflammatory factors such as IL-1 $\beta$, TNF- $\alpha$, high mobility group protein 1 (HMGB1) and other inflammatory mediators, and induce IL-6, which is positively related to the degree of tissue damage. The level of CRP rises accordingly, which triggers the inflammatory process in the brain, produces neurotoxic reactions, affects nerve function, and leads to cognitive impairment ${ }^{[2-3]}$. As the resident immune cells of the central nervous system, microglia can release a variety of factors and compounds with pro-inflammatory, immune- 
modulating and oxidative effects, and are important mediators of central inflammation. Peripheral inflammation can change the morphology of microglia, activate the release of cytokines, and ultimately cause inflammatory factors in the central nervous system, including TNF- $\alpha$, IL-1, IL-6, and certain oxides. And other signaling molecules are produced successively, and are not limited to the hippocampus ${ }^{[4]}$. In addition to the inflammatory response before and after surgery, many damaging changes will also occur during surgery. For example, tissue trauma and wound healing will produce activated oxygen, and the integrity of the blood-brain barrier (BBB) will be imbalanced due to peripheral inflammation, and peripheral activated oxygen stress can induce or aggravate neuroinflammation. Damage to BBB may also induce the release of cytokines and oxidants in the brain. The activation of BBB endothelial cells further aggravates microcirculation dysfunction and leads to impaired cerebral perfusion, which directly affects the occurrence of POD ${ }^{[5]}$.

Advanced age is an independent risk factor for POD. Studies have shown that the occurrence and development of POD is age-dependent, and the brain of elderly patients has shown a low-grade inflammation state at baseline. Agerelated changes in the human brain are characterized by changes in specific areas, such as age-related gray matter volume reduction until the age of 70 , and the annual shrinkage rate is about $2.0 \%$. After 70 years of age, cross-sectional studies have shown that neurodegeneration is accelerated, especially in the hippocampus. The rest, including the prefrontal cortex and white matter, decrease in size with age. The underlying molecular mechanisms of brain aging include changes in calcium signaling, genomic changes, decreased synapses and neurite outgrowth, demyelination, activation of microglia caused by neuroinflammation, changes in cell metabolism, mitochondrial dysfunction, astrocyte hypertrophy, and decreased neuronal activity ${ }^{[6]}$. In summary, as the age increases, the brain exhibits related functional fragility. Due to the fragility of the brain of elderly patients, their brains are more likely to show postoperative delirium and/or cognitive dysfunction after their brains undergo central nervous system inflammation and neuronal damage caused by surgery or anesthesia itself. And postoperative delirium in elderly patients is also easier to develop into permanent cognitive impairment and may even lead to the occurrence of Alzheimer's disease.

Preoperative P.g. infection is an independent risk factor for POD. P.g. itself, antigen components, toxins, metabolites, etc. can invade host tissues and cause tissue damage, such as lipopolysaccharides (LSP) and leukocyte toxins in the outer cell wall membrane. IL can be produced during the host's immune response to Pg and its toxic products. -1 , IL-6, TNF- $\alpha$ cytokines or effector molecules, which in turn cause systemic inflammation. The study of Hu et al. ${ }^{[7]}$ showed that the topical application of P.g.-LPS can induce periodontitis, and cause neuroinflammation by activating the TLR4/NF- $\kappa B$ signaling pathway, which makes SD rats have learning and memory impairment and even over-cognition. decline. A recent meta-analysis explored the relationship between the degree of inflammation and postoperative cognitive dysfunction, and reported that both CRP and IL-6 levels are positively correlated with the prevalence and severity of postoperative cognitive dysfunction. For patients who have been infected with P.g. before surgery, their brains are in an inflammatory state. When they are subjected to a "second blow" to the brain, such as surgery or anesthesia, they are more likely to show postoperative delirium and/or cognitive dysfunction.

Limitations of this study: first, there is no uniform standard for the diagnosis of POD, so there may be some deviations in the diagnosis of POD; second, this study is a single-center study, as a retrospective study, there is insufficient sample size and selection bias; third, the postoperative follow-up of POD is followed up to 3 days after surgery. For patients with POD from 3 days to 1 week after surgery, it may be missed, and the impact on the long-term outcome and prognosis of POD needs further follow-up.

In summary, advanced age and preoperative P.g. infection are independent risk factors for POD. Due to the limited effective prevention and treatment of POD, patients with known high-risk factors before surgery may not be able to completely prevent the occurrence of POD. More research should be carried out in the follow-up to explore the occurrence and prevention of POD, so as to guide the improvement of patients' quality of life and disease outcome after surgery.

\section{References}

[1] Xue Fushan, Zou Yi, Yue Yun. The rename of postoperative cognitive dysfunction and its significant impact [J]. International Journal of Anesthesiology and Resuscitation, 2019, 40(6): 513-515.

[2] Zhu SH, Ji MH, Gao DP, et al. Association between perioperative blood transfusion and early postoperative cognitive dysfunction in aged patients following total hip replacement surgery [J]. Upsala jour med sci, 2014, 119(3): 262 -267.

[3] Takahashi H, Nishibori M. Current status and future prospects in HMGB1 and receptor researches [J]. Nihon Rinsho, 2016, 74(4): 703-711. 
[4] Jin WL, Yang ZY, Xu Y, et al. Microglia in neurodegenerative diseases [J]. Neural Regeneration Research, 2020, 16(2): 270 .

[5] Zhu H, Liu W, Fang H. Inflammation caused by peripheral immune cells across into injured mouse blood brain barrier can worsen postoperative cognitive dysfunction induced by isoflurane [J]. BMC Cell Biology, 2018, 19(1): $251-263$.

[6] Sau R, Veldhuijzen D S, Ottens T H, et al. Association between delirium and cognitive change after cardiac surgery [J]. $B J A, 2017,119(2): 308-315$.

[7] $\mathrm{Hu} \mathrm{Y,} \mathrm{Li} \mathrm{H,} \mathrm{Zhang} \mathrm{J,} \mathrm{et} \mathrm{al.} \mathrm{Periodontitis} \mathrm{Induced} \mathrm{by} \mathrm{P.} \mathrm{gingivalis-LPS} \mathrm{Is} \mathrm{Associated} \mathrm{With} \mathrm{Neuroinflammation} \mathrm{and}$ Learning and Memory Impairment in Sprague-Dawley Rats [J]. Frontiers in Neuroscience, 2020, 14(3): 36-41. 